\title{
Factors Affecting Primary Ionization in Dopant-Assisted Atmospheric Pressure Photoionization (DA-APPI) for LC/MS
}

\author{
Damon B. Robb and Michael W. Blades \\ Department of Chemistry, University of British Columbia, Vancouver, British Columbia, Canada
}

\begin{abstract}
The sensitivity of dopant-assisted atmospheric pressure photoionization (DA-APPI) for LC/MS is generally reduced at higher solvent flow rates. Theory suggests that quenching of excited-state precursors to the dopant ions, via collisions with vaporized solvent molecules, may be one mechanism responsible for this trend. To ascertain if the primary rate of ionization is affected by quenching, experiments were performed utilizing an ionization detector to determine the primary ion current generated by irradiating vaporized mixtures of toluene dopant and methanol solvent. The results indicate that no loss of primary ion current occurs as the solvent flow is increased, provided the dopant-to-solvent ratio is held constant. Additional primary ion current can always be generated by increasing the dopant flow rate and/or the lamp power. Thus, quenching of excited-state precursors to the dopant ions, leading to a reduction in the primary rate of ionization, is not the mechanism responsible for the observed loss of sensitivity at higher liquid solvent flow rates. (J Am Soc Mass Spectrom 2006, 17, 130-138) @ 2006 American Society for Mass Spectrometry
\end{abstract}

$\mathrm{D}$ opant-assisted atmospheric pressure photoionization (DA-APPI) is a new ionization method for LC/MS [1]. Recent review articles describe the method and applications for which it has been utilized [2,3]. One general characteristic of DA-APPI is the sensitivity of the method is diminished as the flow of the solvent delivering the analyte is increased [4-9]. The solvent may affect DA-APPI during the primary photoionization step and/or the ensuing reaction period. In this paper, we examine the effects of the solvent flow, as well as the dopant flow and lamp power, on the primary photoionization step in DA-APPI.

As a starting point for the study of the photoionization process in DA-APPI, it is instructive to consider the literature of photoionization detectors (PIDs), since in both methods the same mechanisms are involved in generating the primary photoions. A number of researchers have published theory describing the performance of PIDs [10-12]. In the last of these reports, DeWit and Jorgenson described factors affecting the photoionization efficiency of a PID coupled with a liquid chromatograph. As part of their theory, the effects of the solvent in the vapor were considered. The following theoretical analysis is an extended adaptation of the theory of DeWit and Jorgenson [12].

Published online January 18, 2006

Address reprint requests to Dr. M. W. Blades, Department of Chemistry, University of British Columbia, 2036 Main Mall, Vancouver V6T 1Z1, B.C., Canada. E-mail: blades@chem.ubc.ca

\section{Theory}

In DA-APPI, after vaporization of the liquid sample stream and dopant, the vapor mixture is irradiated with VUV photons, normally from a krypton discharge lamp $(\lambda=1236$ and $1165 \AA ; \mathrm{h} \nu=10.03$ and $10.64 \mathrm{eV}$ [13]). The dopant (D) is selected so that it has an ionization energy (IE) less than $\mathrm{h} \nu$, and usually the solvent (S) has IE $>\mathrm{h} \nu$. Irradiation of the vapor may result in direct photoionization of the dopant (eq 1), as well as photoexcitation of the dopant and the solvent (eqs 2 and 3 ):

$$
\begin{aligned}
& \mathrm{D}+\mathrm{h} \nu \rightarrow \mathrm{D}^{+\cdot}+\mathrm{e}^{-} \\
& \mathrm{D}+\mathrm{h} \nu \rightarrow \mathrm{D}^{*} \\
& \mathrm{D}+\mathrm{h} \nu \rightarrow \mathrm{S}^{*}
\end{aligned}
$$

The nitrogen carrier gas is essentially transparent to photons at wavelengths of 1236 and $1165 \AA$ [14]. The analyte, solvent additives, and impurities are assumed to be at concentrations low enough that their absorption of the photons can be ignored.

The light intensity at a distance $x(\mathrm{~cm})$ from the lamp window after absorption by both the dopant and solvent is given by the Beer-Lambert law:

$$
I_{x}=I_{0} \mathrm{e}^{-x\left(\sigma_{D} n_{D}+\sigma_{S} n_{S}\right)}
$$

where $I_{x}=$ light intensity at distance $x$ (photons $\left.\mathrm{s}^{-1}\right), I_{0}$ $=$ light intensity emanating from the lamp (photons $\left.\mathrm{s}^{-1}\right), \sigma_{D}=$ absorption cross-section for $\mathrm{D}(\mathrm{Mb}, 1 \mathrm{Mb}=$ 
$\left.10^{-18} \mathrm{~cm}^{2}\right), n_{D}=$ number density of $\mathrm{D}\left(\mathrm{cm}^{-3}\right), \sigma_{S}=$ absorption cross-section for $S(\mathrm{Mb})$, and $n_{S}=$ number density of $\mathrm{S}\left(\mathrm{cm}^{-3}\right)$.

The light intensity absorbed by the dopant, $I_{D}$ (photons $\mathrm{s}^{-1}$ ), over infinitesimal distance $\mathrm{d} x$ from $x$ to $x+\mathrm{d} x$ is

$$
I_{D}=I_{x}\left(1-\mathrm{e}^{-\sigma_{D} n_{D} \mathrm{~d} x}\right)
$$

By applying the first two terms of the exponential expansion, a simplified expression is obtained:

$$
I_{D}=I_{x} \sigma_{D} n_{D} \mathrm{~d} x
$$

Substituting eq 4 into eq 6 gives

$$
I_{D}=I_{0} \sigma_{D} n_{D} \mathrm{~d} x \cdot \mathrm{e}^{-x\left(\sigma_{D} n_{D}+\sigma_{S} n_{S}\right)}
$$

Integration from 0 to $L$, where $L$ is the path length of the radiation through the absorbing medium, yields

$$
I_{D}=I_{0} \frac{\sigma_{D} n_{D}}{\sigma_{D} n_{D}+\sigma_{S} n_{S}}\left[1-\mathrm{e}^{-L\left(\sigma_{D} n_{D}+\sigma_{S} n_{S}\right)}\right]
$$

To convert the expression for the rate of photon absorption by the dopant to the rate of dopant ion creation, a variety of processes following the absorption event must be considered. The first process that affects the overall rate of ionization is direct photoionization of the dopant (eq 1), characterized by an efficiency $\phi_{1}$ (ions photon $\left.^{-1}\right)$ :

$$
\left(\frac{\mathrm{d} D^{+\cdot}}{\mathrm{d} t}\right)_{1}=\phi_{1} \cdot I_{D}
$$

The remaining processes all involve the excited-state intermediate $\mathrm{D}^{*}$ and will be considered together.

The events that may be expected to occur following the formation of $\mathrm{D}^{*}$ include

$$
\begin{array}{llll}
\text { RXN 1 } & \mathrm{D}^{*} \rightarrow \mathrm{A}+\mathrm{B} & \text { predissociation } & R_{1}=k_{1} \cdot n_{D^{*}} \\
\text { RXN 2 } & \mathrm{D}^{*} \rightarrow \mathrm{D}+\mathrm{hv} & \text { fluorescence } & R_{2}=k_{2} \cdot n_{D^{*}} \\
\text { RXN 3 } & \mathrm{D}^{*}+\mathrm{S} \rightarrow \mathrm{D}+\mathrm{S} & \text { collisional quenching } & R_{3}=k_{3} \cdot n_{D^{*}} \cdot n_{S} \\
\text { RXN 4 } & \mathrm{D}^{*}+\mathrm{N}_{2} \rightarrow \mathrm{D}+\mathrm{N}_{2} & \text { collisional quenching } & R_{4}=k_{4} \cdot n_{D^{*}} \cdot n_{N_{2}} \\
\text { RXN 5 } & \mathrm{D}^{*} \rightarrow \mathrm{D}^{+\cdot}+\mathrm{e}^{-} & \text {autoionization } & R_{5}=k_{5} \cdot n_{D^{*}}
\end{array}
$$

where $\mathrm{A}$ and $\mathrm{B}$ are neutral fragments of $\mathrm{D}, R_{n}$ and $k_{n}$ are the reaction $(R X N)$ rate and the rate constant for reaction $\mathrm{n}$, respectively, $n_{D^{*}}=$ number density of $\mathrm{D}^{*}\left(\mathrm{~cm}^{-3}\right)$, and $n_{N_{2}}=$ number density of nitrogen $\left(\mathrm{cm}^{-3}\right)$. From the ratio of the rate of autoionization to the combined rates of these five processes, a second term for ionization efficiency, $\phi_{2}$, can be expressed in the following manner:

$$
\phi_{2}=\frac{k_{5}}{k_{1}+k_{2}+k_{3} \cdot n_{S}+k_{4} \cdot n_{N_{2}}+k_{5}}
$$

The rate of indirect dopant ion production through autoionization is then given by

$$
\left(\frac{\mathrm{d} D^{+\cdot}}{\mathrm{d} t}\right)_{2}=\left(1-\phi_{1}\right) \cdot \phi_{2} \cdot I_{D}
$$

where the factor $\left(1-\phi_{1}\right)$ accounts for the absorbed light intensity that results in direct photoionization.

Combining the expressions for direct and indirect ionization, eqs 9 and 11, the total primary rate of dopant ionization, $R_{P}$ (ions $\mathrm{s}^{-1}$ ), can then be expressed as

$$
R_{P}=\left(\frac{\mathrm{d} D^{+\cdot}}{\mathrm{d} t}\right)_{1}+\left(\frac{\mathrm{d} D^{+\cdot}}{\mathrm{d} t}\right)_{2}=\left[\phi_{1}+\left(1-\phi_{1}\right) \cdot \phi_{2}\right] \cdot I_{D}
$$

or, introducing a term, $\phi_{T}$ (ions photon $^{-1}$ ), for the total quantum yield of ionization

$$
\phi_{T}=\phi_{1}+\left(1-\phi_{1}\right) \cdot \phi_{2}
$$

so that

$$
R_{P}=\phi_{T} \cdot I_{D}
$$

\section{Summary}

Theory suggests that the primary rate of dopant ionization, $R_{P}$, may be affected by the solvent flow rate through two mechanisms. Eq 8 indicates that, for a given dopant flow rate, a reduction in the light intensity absorbed by the dopant, $I_{D}$, is expected as the solvent flow is increased. This is because the solvent absorbs the photons, too, attenuating the light intensity available for absorption by the dopant. However, eq 8 also indicates that the diminishment of $I_{D}$ with increased solvent flow can be prevented by maintaining a constant ratio of dopant-to-solvent in the vapor. Hence, $I_{D}$ can be made independent of the solvent flow rate, and no diminishment in $R_{P}$ - because of absorption of photons by the solvent-need accompany increases in solvent flow. On the other hand, eq 10 indicates that quenching of excited-state intermediates, $\mathrm{D}^{*}$, via collisions with solvent molecules, may lower the autoionization efficiency, $\phi_{2}$, regardless of the amount of dopant added. Since the rate of collisional quenching is expected to scale directly with the number density of solvent in the vapor, $\phi_{2}$ may continuously decrease as the solvent flow is increased. If the direct ionization efficiency, $\phi_{1}$, is small, then it is conceivable that collisional quenching at high solvent flow rates may result in a significant reduction of the total quantum yield of ionization, $\phi_{T}$. Thus, theory suggests that $R_{P}$ may be diminished as the solvent flow is increased, even when $I_{D}$ is held constant by maintaining a fixed ratio of dopant-to-solvent.

We have constructed an ionization detector for determining the primary ion current generated by irradiating vaporized mixtures of dopant and solvent. Experiments have been performed to probe the effects of solvent flow, dopant flow, and lamp power on the primary rate of dopant ionization in DA-APPI, and to 


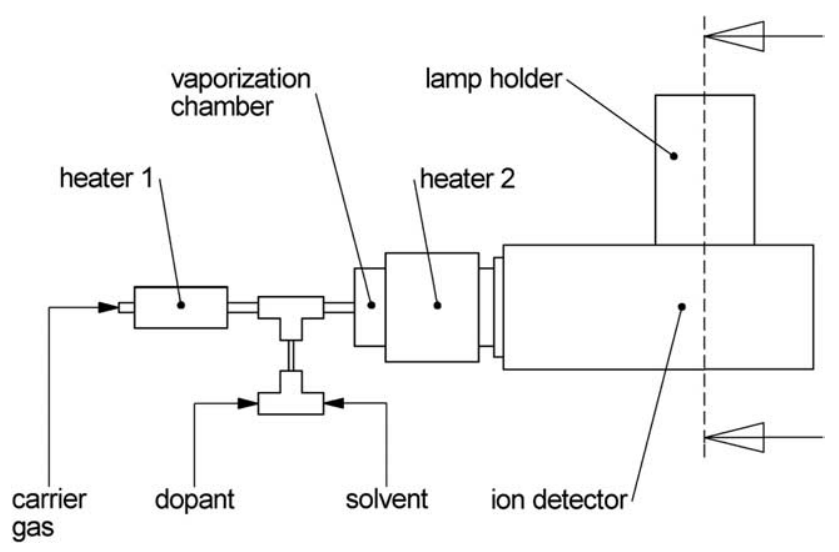

Figure 1. Schematic of the experimental apparatus (top view) showing the arrangement of the vaporizer assembly, the ionization detector, and the lamp. The dashed line indicates the location of the vertical plane, and the arrows indicate the perspective, of the cross-section displayed in Figure 2.

ascertain, specifically, if the rate of dopant ionization is indeed reduced by solvent-induced collisional quenching.

\section{Experimental}

\section{Chemicals}

The test system utilized toluene (IE $=8.83 \mathrm{eV}$ ) as the dopant and methanol (IE $=10.84 \mathrm{eV}$ ) as the solvent. Methanol and toluene were HPLC grade, and were used as received from Fisher Scientific (Ottawa, Ontario, Canada). Nitrogen (IE $=15.58 \mathrm{eV}$ ) was used for both the carrier and lamp gases. The nitrogen was pre-purified grade $\left(99.998 \%, \mathrm{O}_{2}<5 \mathrm{ppm}, \mathrm{H}_{2} \mathrm{O}<3\right.$ ppm) from Praxair (Mississauga, Ontario, Canada). (All ionization energies are from reference [15].)

\section{Apparatus}

Figure 1 is a schematic of the experimental apparatus, illustrating the arrangement of the vaporizer assembly, the ionization detector, and the lamp. The first component of the vaporizer assembly was heater 1 , a $6 \mathrm{~cm}$ length of copper tubing (o.d. $=1 / 8$ in, i.d. $=5 / 64$ in) wrapped with nichrome resistance wire, used to preheat the carrier gas. Power to heater 1 was supplied from a variable transformer. The volumetric flow rate of the carrier gas was $3.01 \mathrm{~min}^{-1}$ (at STP), set with a Model 810C mass flow controller from Sierra Instruments (Monterey, CA). The preheated carrier gas flowed into a brass union tee (o.d. $=1 / 8$ in) where it was mixed with the liquid solvent and dopant. Glass wool was placed in this mixing tee, to increase the surface area of the liquid as it was delivered, via capillary action, and thereby stabilize the vaporization process. Solvent and dopant were supplied by separate syringe pumps from Harvard Apparatus (Holliston, MA) and mixed in another tee (o.d. $=1 / 16$ in) before delivery into the vaporizer assembly. The power to heater 1 was adjusted as necessary to ensure that the carrier gas immediately and completely vaporized the liquid mixture as it was delivered, without sputtering. The resulting vapor flowed through a secondary vaporization chamber (a stainless-steel cylinder with i.d. $=1.5 \mathrm{~cm}$ and length $=$ $4.0 \mathrm{~cm}$ ), maintained at a constant temperature of $200^{\circ} \mathrm{C}$ by a band heater (heater 2), a thermocouple, and a model 6100 temperature controller from Omega (Stamford, CT). The second heating stage ensured that the temperature of the vapor mixture as it entered the ionization detector was unchanged $\left( \pm 5^{\circ} \mathrm{C}\right)$ by variations in the solvent and dopant flow rates.

Figure 2 shows a cross-section of the ionization detector as well as the associated electronics. The housing of the ionization detector was a Teflon block, with a rectangular-cross-section main channel for the vapor

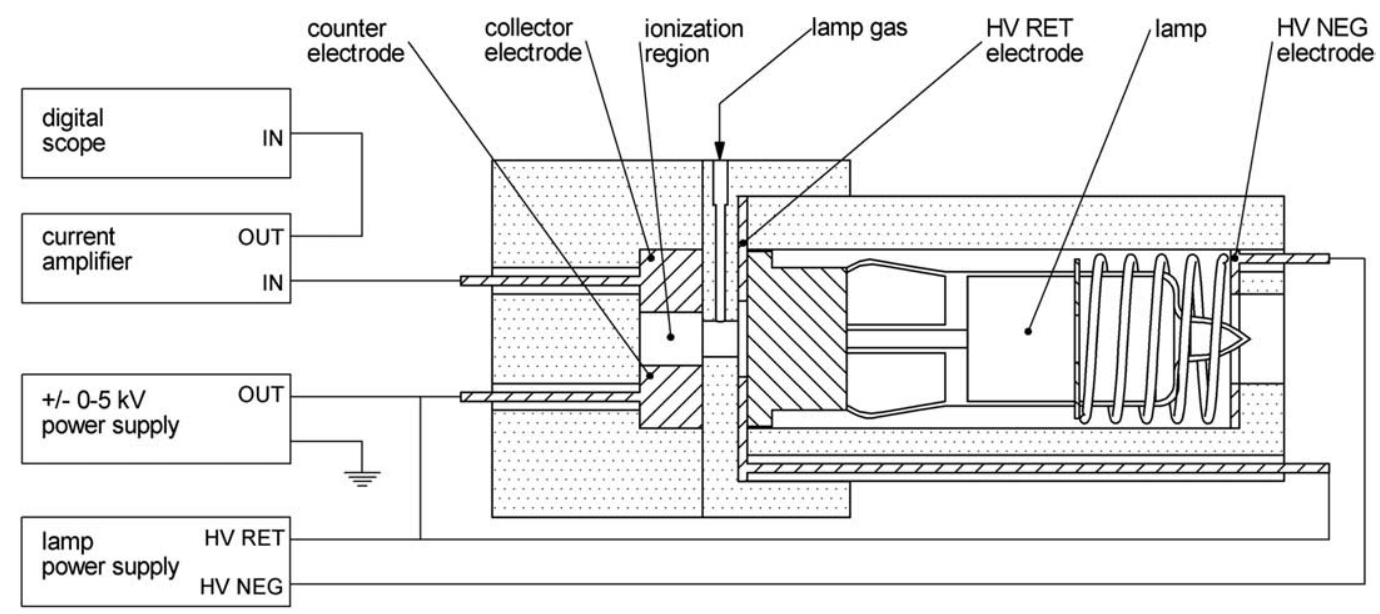

Figure 2. Schematic of the ionization detector showing a cross-section through the center of the ionization region of the main channel, and the associated electronics. The perspective is from the end of the ionization detector, looking down the main channel towards the vaporizer assembly. 
mixture. The main channel was bordered along its 7.0 $\mathrm{cm}$ length by two opposite-facing stainless-steel electrodes. These electrodes were $0.7 \mathrm{~cm}$ wide and separated by a distance of $0.6 \mathrm{~cm}$. The photoionization lamp was a model PKS 100 krypton discharge lamp from Cathodeon Ltd. (Cambridge, England). The lamp and its Teflon holder were situated in a side-bore of the housing, halfway along the length of the main channel, so that the center of the main channel was irradiated, forming an ionization region. A side-channel (diameter $=0.4 \mathrm{~cm}$, depth $=0.4 \mathrm{~cm}$ ) between the main channel and the lamp served as an aperture, so that the electrodes bordering the ionization region were not irradiated. A flow of nitrogen was introduced directly in front of the lamp to exclude the solvent-and-dopantcontaining vapor from the side-channel. This step was to prevent photoionization of the dopant from occurring within the side-channel, where the detection efficiency for charged species was low, and also to protect the lamp's window. The flow rate of the lamp gas was $1.01 \mathrm{~min}^{-1}$ (at STP), set with another model 810C mass flow controller from Sierra Instruments. In control experiments, the temperature of the vapor in the ionization region, after mixing of the main vapor and the lamp gas, was measured to be about $110^{\circ} \mathrm{C}$; this value varied by less than $\pm 5^{\circ} \mathrm{C}$ over the range of dopant and solvent flow rates. The entire vapor flow vented to atmosphere, without obstruction, so that there was no significant pressure build up within the ionization detector.

The two electrodes bordering the main channel and the ionization region were the counter electrode and the collector electrode. The collection voltage, $V(\mathrm{~V})$, is defined as the absolute value of the potential difference between the two. The potential of the counter electrode was set by a model PS350 high voltage power supply from Stanford Research Systems (Sunnyvale, CA). The collector electrode was at virtual ground potential, as it was connected to ground through the input of a model 427 current amplifier from Kiethley Instruments (Cleveland, $\mathrm{OH})$. Measurements of the current, $i(\mathrm{~A})$, resulting from charged species discharging at the collector electrode were made via the current amplifier and a model TDS 340A digital oscilloscope from Tektronix (Wilsonville, OR). The gain on the current amplifier was $10^{6} \mathrm{~V}$ $\mathrm{A}^{-1}$ and the rise time was $300 \mathrm{~ms}$. Each experimental datum presented here is the mean of 1000 samples acquired on the oscilloscope over a $50 \mathrm{~s}$ period. The error bars provided correspond to $\pm 2 s$, where $s$ is the standard deviation.

The lamp was powered by a custom HV supply (Electronic Engineering Services, Chemistry Department, University of British Columbia) that allowed the lamp current to be varied between 0.2 and $2 \mathrm{~mA}$, in steps of $0.2 \mathrm{~mA}$. The lamp power supply was floated at the potential of the ionization detector's counter electrode. Electrical contact to the anode at the base of the lamp (HV RET, at the potential of the counter electrode) was made via a washer-shaped electrode that abutted

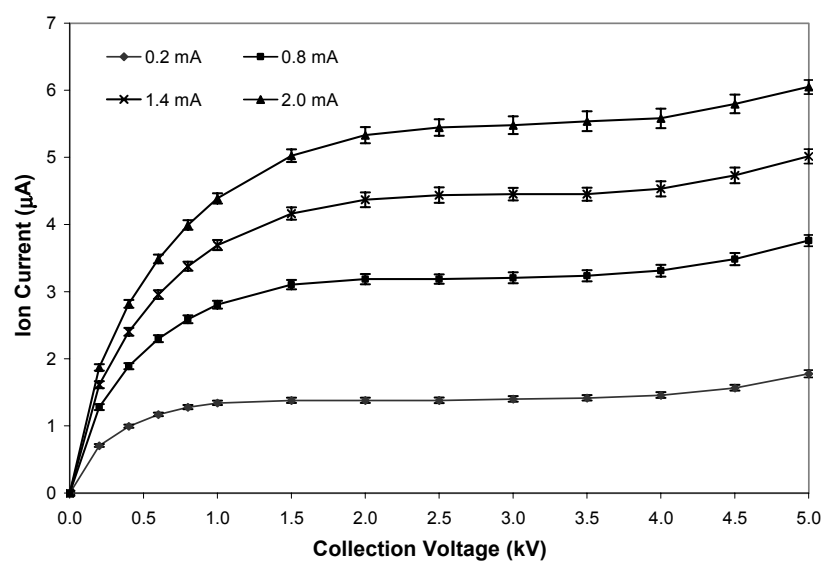

Figure 3. Plots of ion current versus collection voltage for several lamp current settings. A negative voltage was applied to the counter electrode of the ionization detector so the measured current was attributable to negative charge carriers, primarily electrons. The indicated ion current was taken from the measured electron current, since the rates of positive ion and electron generation are equal. The toluene dopant flow rate was $100 \mu \mathrm{l}$ $\mathrm{min}^{-1}$ and no methanol solvent was added.

the side-channel of the ionization detector. Electrical contact to the lamp's cathode (HV NEG) was made via a spring contact to another washer-shaped electrode at the back of the lamp holder.

\section{Method}

Preliminary experiments were performed to find the collection voltage required to determine the saturation current, $i_{S A T}(\mathrm{~A})$. The saturation current is the current that results when all the charge carriers of one polarity are collected, as they are generated, without loss [16, 17]. Knowledge of $i_{S A T}$ allows for the determination of the primary rate of ionization, since

$$
i_{S A T}=R_{P} \cdot e
$$

where $e$ is the elementary charge. Generally, a higher $V$ is required to determine $i_{S A T}$ as the rate of ionization is increased, due to the accompanying increased rate of recombination, and also plasma effects (e.g., Debye shielding). For all the results presented here, the photoelectron current was measured, rather than the equivalent photoion current, because $i_{S A T}$ for the electrons could be measured at lower $V$, presumably because of their much higher mobility. This enabled greater ionization rates to be determined accurately. (Note that experimental photoion currents are referred to in the remainder of the paper, since this is the subject of discussion, even though the actual measured property was the photoelectron current.) Figure 3 shows plots of $i$ versus $V$ that were used to determine the optimum $V$ for subsequent experiments (see the figure caption for experimental details). These plots contain three regions [17]: (1) at too low $V, \mathrm{~d} i / \mathrm{d} V>0$ and $i<i_{S A T}$, due to incomplete 


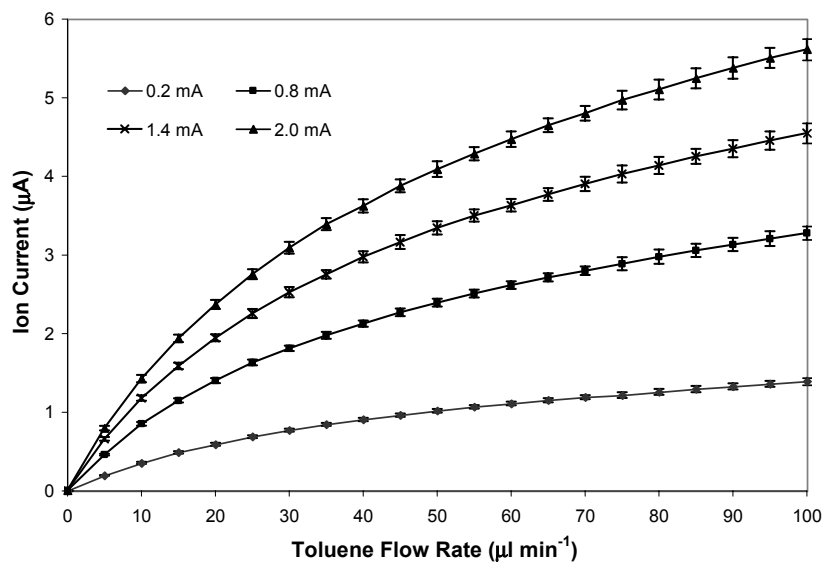

Figure 4. Plots of ion current versus toluene dopant flow rate, for several lamp current settings. No methanol solvent was added.

charge collection; (2) in the optimum $V$ range, $\mathrm{d} i / \mathrm{d} V$ $=0$ and $i=i_{S A T}$, due to complete charge collection; (3) at too high $V, \mathrm{~d} i / \mathrm{d} V>0$ and $i>i_{S A T}$, due to charge amplification (electrical breakdown). In Figure 3 , the region of incomplete charge collection extends to about $2 \mathrm{kV}$ for the highest lamp current setting (and thus the highest ionization rate), while in all the plots the beginnings of charge amplification are evident above about $4 \mathrm{kV}$. Accordingly, a fixed collection voltage of (negative) $3.0 \mathrm{kV}$ was used to obtain the results presented in this paper.

\section{Results and Discussion}

\section{Dopant Only}

We first examine the primary rate of ionization in the absence of solvent and its dependence on the toluene dopant flow rate and lamp current. Figure 4 shows plots of primary ion current (i.e., $i_{S A T}$ ) versus toluene flow rate for several lamp current settings. In each plot, the ion current is negligible when the toluene flow is zero (indicating that the rate of photoelectron generation from the carrier gas and the surfaces of the ionization detector is negligible). As the toluene flow is increased, the ion current generally increases. Regarding the effect of the lamp current, for each toluene flow rate the total percentage increase in the ion current upon raising the lamp current from 0.2 to $2.0 \mathrm{~mA}$ is about the same, $\sim 300 \%$. We deduce from this result that the corresponding increase in the lamp's emission intensity, $I_{0}$, is also $\sim 300 \%$, since $i_{S A T}$ and $I_{0}$ are proportional-see eqs 8,14 , and 15 . The data then confirm that $I_{0}$ increases with lamp current, though the relationship is nonlinear and diminishing returns in emission intensity are obtained as the lamp is driven at higher power. Regarding the attainable primary ionization rate, $R_{P}$, the highest ion current generated in this experiment, 5.6 $\mu \mathrm{A}$, is equivalent to $3.5 \times 10^{13}$ ions $\mathrm{s}^{-1}$. This level of reagent ion production is in the same range as that commonly utilized in corona discharge atmospheric pressure chemical ionization (APCI) sources [18, 19], so we know that DA-APPI sources are not inherently dim. Thus, the results in Figure 4 demonstrate that $R_{P}$ can be increased by raising the dopant flow and/or the lamp current, and also that a quite high $R_{P}$ can be had using a conventional PID lamp and a modest dopant flow, at least in the absence of solvent.

We now take a closer look at the effect of the toluene dopant flow rate on the primary rate of ionization. In Figure 4, the slope of each plot is initially regressive, continuously decreasing as the toluene flow is increased, up until about $50 \mu \mathrm{l} \mathrm{min}{ }^{-1}$. At higher flow rates, however, each slope stabilizes. Linear trend lines, each with $R^{2}>0.993$, may be fit to the data for flow rates of 50 to $100 \mu \mathrm{l} \mathrm{min}^{-1}$ (results not shown). The relationship between ion current and toluene flow above $50 \mu \mathrm{min}^{-1}$ is clearly different from that below. When the data from Figure 4 are normalized to the ion currents at the highest toluene flow, the resulting plots overlap perfectly (within experimental error; results not shown), indicating that the two distinct trends in ion current with increases in toluene flow are not affected by the intensity of the lamp or the magnitude of the ionization rate at each flow. We take this as evidence that the trends in the data reflect the actual trends in the ionization rate, and that the results are not affected by space-charge- or plasma-related anomalies of the measurement process.

To check the experimental results against theory, it is desirable to calculate $i_{S A T}$ as a function of the dopant flow rate. The saturation current is calculable from eqs 8,14 , and 15, provided the following are known: for each of the two ionizing wavelengths, the intensity of the lamp, $I_{0}$, as well as the absorption cross-section, $\sigma_{D}$, and the total quantum yield of ionization, $\phi_{T}$, of the toluene dopant. The path length, $L$, of the radiation through the absorbing medium must also be known, as well as the number density of the dopant, $n_{D}$. Assuming ideal gas behavior and no local anisotropy, $n_{D}$ can be calculated from the liquid toluene flow rate, the sum of the carrier and lamp gas flow rates, and the temperature of the vapor. The path length is taken to be the width of the main channel of the ionization detector (i.e., $L \approx 0.7 \mathrm{~cm}$ ). Regarding absorption cross-section data for toluene, these are presented as a function of wavelength in Figures 3 and 4 of reference [20], from which we extract $\sigma_{D} \approx 48$ and $64 \mathrm{Mb}$, respectively, for $\lambda=1236$ and $1165 \AA$. Now, however, neither $I_{0}$ for the lamp nor $\phi_{T}$ for toluene is known to us, so direct calculations of $i_{S A T}$ are precluded. Nevertheless, the ratio of intensities at 1236 and $1165 \AA$ is provided by the lamp's manufacturer, $\sim 4: 1$ [13], allowing us to determine a weighted-average cross-section for toluene, $\sigma_{D} \approx 51 \mathrm{Mb}$. This in turn enables the calculation of $I_{D} / I_{0}$, which is proportional to $i_{S A T}$, as we shall now show. Substituting eq 14 into eq 15 and multiplying by $I_{0} / I_{0}$ yields 


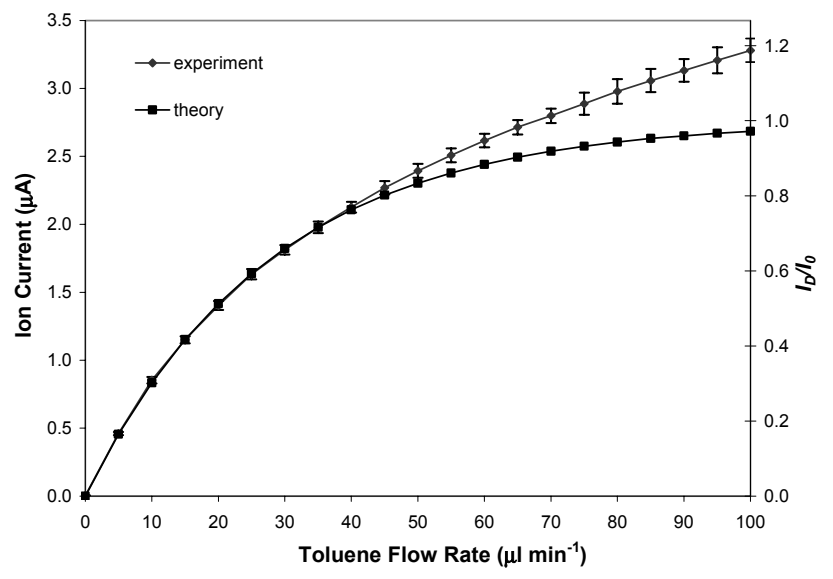

Figure 5. Plots of ion current (experiment) and calculated $I_{D} / I_{0}$ (theory) versus toluene dopant flow rate. No methanol solvent was added. For the experimental data, lamp current $=0.8 \mathrm{~mA}$. The scaling factor, $C$, for the two vertical axes is $2.76 \mu \mathrm{A}$.

$$
i_{S A T}=\left(\frac{I_{D}}{I_{0}}\right) \cdot I_{0} \cdot \phi_{T} \cdot e=\left(\frac{I_{D}}{I_{0}}\right) \cdot C
$$

where $C(\mathrm{~A})$ is equal to $I_{0} \cdot \phi_{T} \cdot e$. For a given lamp current, $C$ is expected to be constant under dopant-only conditions, since $I_{0}$ is affected only by the lamp current and theory (above) predicts that $\phi_{T}$ is independent of the toluene flow. Thus, by fitting experimental $i_{S A T}$ data to calculated values of $I_{D} / I_{0}$, we can compare the trends in the experimental and theoretical results.

Figure 5 displays plots of experimental ion current and calculated $I_{D} / I_{0}$ versus toluene flow rate. The experimental data were acquired with lamp current $=0.8$ $\mathrm{mA}$. The two vertical axes of the figure have been scaled so that ion current $\left(i_{S A T}\right)$ is equal to $I_{D} / I_{0}$ times $2.76 \mu \mathrm{A}$. This scaling factor corresponds to $C$ from eq 16, so each theoretical datum can be read from either axis, as the calculated $I_{D} / I_{0}$ value or the equivalent ion current. This value of $C$ was selected because it provides the best fit to the experimental and theoretical data at the lower toluene flow rates, where the slopes of both plots are initially regressive and the experimental results appear to conform with theory. No scaling factor can accurately fit the trends in the two plots at higher toluene flows. This is because at toluene flows above about $50 \mu \mathrm{l}$ $\mathrm{min}^{-1}$ the slope of the experimental plot stabilizes, as noted above, while the slope of the theoretical plot remains regressive, as $I_{D} / I_{0}$ asymptotically approaches unity. Current theory cannot account for the observed near-linear relationship between ion current and toluene flow at the higher toluene flow rates. Rather than arbitrarily force the two plots to an average, poor fit over the entire range of toluene flows, we speculate that current theory accurately describes the relevant physical processes at the lower toluene flow rates-where the match between the experimental and theoretical trends is remarkably close-and that an additional, previously ignored process becomes significant at the higher tolu- ene flows. Specifically, we propose that Penning ionization of the dopant via excited-state intermediates, $\mathrm{D}^{*}$, may be occurring when $n_{D}$ is large:

$$
\text { RXN } 6 \mathrm{D}^{*}+\mathrm{D} \rightarrow \mathrm{D}+\mathrm{D}^{+\cdot}+\mathrm{e}^{-} \text {Penning ionization } R_{6}=k_{6} \cdot n_{D^{*}} \cdot n_{D}
$$

The expression for the efficiency of indirect ionization, $\phi_{2}$, now for both auto- and Penning ionization, then becomes

$$
\phi_{2}=\frac{k_{5}+k_{6} \cdot n_{D}}{k_{1}+k_{2}+k_{3} \cdot n_{S}+k_{4} \cdot n_{N_{2}}+k_{5}+k_{6} \cdot n_{D}}
$$

Eq 17 indicates that $\phi_{2}$, and thus $\phi_{T}$, may be an increasing function of the toluene dopant flow rate. This explains how the primary rate of ionization may still increase with dopant flow, even when $I_{D} / I_{0}$ is relatively static because it is near unity.

\section{Dopant and Solvent}

To study the effects of the solvent on $R_{P}$, the ion detector was used to determine primary ion current as a function of the dopant and solvent flow rates. Figure 6 shows plots of ion current versus toluene flow rate for several lamp current settings, obtained with a fixed methanol solvent flow of $500 \mu 1 \mathrm{~min}^{-1}$. As in Figure 4, the ion current in the absence of toluene is negligible (indicating that the rate of photoelectron generation from methanol alone is negligible). For each lamp current setting, the ion current again increases continuously with toluene flow. Unlike the dopant-only case, there is very little curvature in the plots over the entire range of toluene flows. The ion current at each toluene flow is again proportional to the lamp current. With the solvent, however, the ion current for a given toluene flow and lamp current is lower than in the absence of solvent. For example, with the lamp current fixed at 2.0 $\mathrm{mA}$, toluene flow $=100 \mu \mathrm{l} \mathrm{min}{ }^{-1}$ yielded $5.6 \mu \mathrm{A}$ in the

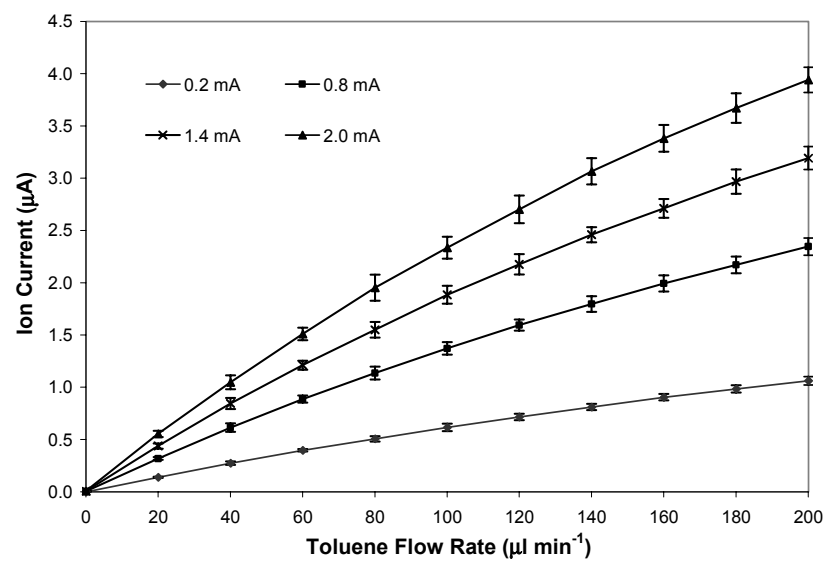

Figure 6. Plots of ion current versus toluene dopant flow rate, for several lamp current settings. The methanol solvent flow rate was $500 \mu 1 \mathrm{~min}^{-1}$. 


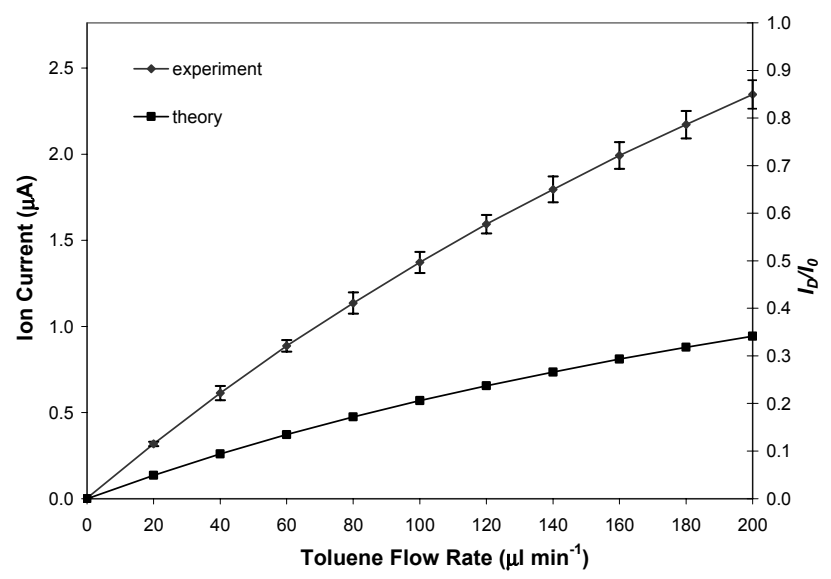

Figure 7. Plots of ion current (experiment) and calculated $I_{D} / I_{0}$ (theory) versus toluene dopant flow rate. The methanol solvent flow rate was $500 \mu \mathrm{l} \mathrm{min}{ }^{-1}$. For the experimental data, lamp current $=0.8 \mathrm{~mA}$. The scaling factor, $C$, for the two vertical axes is $2.76 \mu \mathrm{A}$.

absence of solvent, but only $2.3 \mu \mathrm{A}$ with methanol flow $=500 \mu \mathrm{l} \mathrm{min}{ }^{-1}$. This demonstrates that the solvent does indeed have a negative effect on the primary rate of dopant ionization. Nevertheless, the results in Figure 6 indicate that additional primary ion current can always be generated by increasing the dopant flow and/or the lamp current. Primary ion currents in excess of $4 \mu \mathrm{A}$ are easily generated, even with the relatively high solvent flow of $500 \mu \mathrm{l} \mathrm{min}{ }^{-1}$, so it does not appear that the solvent flow limits $R_{P}$.

It is also possible to calculate $I_{D} / I_{0}$ when solvent is present (eq 8), so that experiment and theory can be compared for the dopant-and-solvent case. The requisite absorption cross-section data for methanol are presented in reference [21], from which we extract $\sigma_{S} \approx$ $15 \mathrm{Mb}$ at each of the ionizing wavelengths. Figure 7 displays plots of experimental ion current and calculated $I_{D} / I_{0}$ versus toluene flow rate, for methanol flow $=$ $500 \mu \mathrm{l} \mathrm{min}{ }^{-1}$. The experimental data were again acquired with lamp current $=0.8 \mathrm{~mA}$, enabling these results to be compared with those of Figure 5 . The scaling factor for the two vertical axes is the same as that used in Figure 5. Assuming for the moment that the solvent acts only as a photon sink (i.e., that it only affects $\left.I_{D} / I_{0}\right)$, and that $\phi_{T}$ is essentially independent of the toluene flow when it is below $50 \mu \mathrm{l} \mathrm{min} \mathrm{m}^{-1}$, as proposed above, we then expect the experimental and theoretical plots of Figure 7 to coincide at toluene flows below $50 \mu \mathrm{l} \mathrm{min}{ }^{-1}$. The first assumption also leads us to expect the experimental plot to rise above the theoretical plot at higher toluene flows, since $\phi_{T}$ appears to be an increasing function of $n_{D}$, as we have discussed, while the scaling factor for the two plots is fixed at the value believed to be accurate for the lower flows. On the other hand, in the event that solvent-quenching of $\mathrm{D}^{*}$ is indeed a significant process, causing $\phi_{T}$ to be substantially reduced by the solvent, we expect the experimental plot to be below the theoretical plot across the entire range of toluene flows. The actual result is quite different from these scenarios: for each toluene flow, the experimental ion current is more than twice the theoretical equivalent (the product of $I_{D} / I_{0}$ and $C$ ), even at

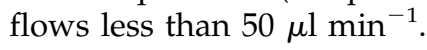

One possible explanation for the result in Figure 7 is that the reported value of $\sigma_{S}$ is too high, leading to a systematic underestimation of $I_{D} / I_{0}$. However, if this were true, then the solvent would have little impact on $I_{D} / I_{0}$, and there should be curvature in the experimental plot, as seen in the dopant-only case. Both plots in Figure 7 are nearly linear, though the magnitudes of their slopes differ. The near-linearity of the theoretical plot is a consequence of the essentially complete absorption of the photons by the dopant and solvent; i.e., from eq 8 ,

$$
\mathrm{e}^{-L\left(\sigma_{D} n_{D}+\sigma_{S} n_{S}\right)} \ll 1
$$

so that

$$
I_{D} / I_{0} \approx \frac{\sigma_{D} n_{D}}{\sigma_{D} n_{D}+\sigma_{S} n_{S}}
$$

Eq 19 indicates how $I_{D} / I_{0}$ increases in a near-linear fashion when the dopant flow is raised, when the solvent's flow and absorption cross-section are substantial. The shape of the experimental plot in Figure 7 is then in accordance with the calculations of $I_{D} / I_{0}$, though the actual ionization rates are higher than expected.

Another explanation for the deviation of the experimental results from theory is that the solvent acts as more than a simple photon sink, as previously assumed. We speculate that excited-state intermediates, $\mathrm{S}^{*}$, resulting from absorption of the photons by the solvent (eq 3), may also lead to Penning ionization of the dopant; i.e.

RXN $7 \mathrm{~S}^{*}+\mathrm{D} \rightarrow \mathrm{S}+\mathrm{D}^{+\cdot}+\mathrm{e}^{-} \quad$ Penning ionization $R_{7}=k_{7} \cdot n_{S^{*}} \cdot n_{D}$

To update theory to include the contribution of reaction 7 to the total primary ionization rate, we must first consider the rate of photon absorption by the solvent, $I_{S}$ (photons s ${ }^{-1}$ ):

$$
I_{S}=I_{0} \frac{\sigma_{S} n_{S}}{\sigma_{D} n_{D}+\sigma_{S} n_{S}}\left[1-\mathrm{e}^{-L\left(\sigma_{D} n_{D}+\sigma_{S} n_{S}\right)}\right]
$$

Then, we must consider the events besides Penning ionization that may be expected to occur following the formation of $S^{*}$ :

$$
\begin{array}{llll}
\mathrm{RXN} 8 & \mathrm{~S}^{*} \rightarrow \mathrm{A}^{\prime}+\mathrm{B}^{\prime} & \text { predissociation } & R_{8}=k_{8} \cdot n_{S^{*}} \\
\mathrm{RXN} 9 & \mathrm{~S}^{*} \rightarrow \mathrm{S}+\mathrm{h} \nu & \text { fluorescence } & R_{9}=k_{9} \cdot n_{S^{*}} \\
\mathrm{RXN} 10 & \mathrm{~S}^{*}+\mathrm{S} \rightarrow \mathrm{S}+\mathrm{S} & \text { collisional quenching } & R_{10}=k_{10} \cdot n_{S^{*}} \cdot n_{S} \\
\mathrm{RXN} 11 & \mathrm{~S}^{*}+\mathrm{N}_{2} \rightarrow \mathrm{S}+\mathrm{N}_{2} & \text { collisional quenching } & R_{11}=k_{11} \cdot n_{S^{*}} \cdot n_{N_{2}}
\end{array}
$$

where $\mathrm{A}^{\prime}$ and $\mathrm{B}^{\prime}$ are neutral fragments of $\mathrm{S}$, and $n_{S^{*}}=$ 


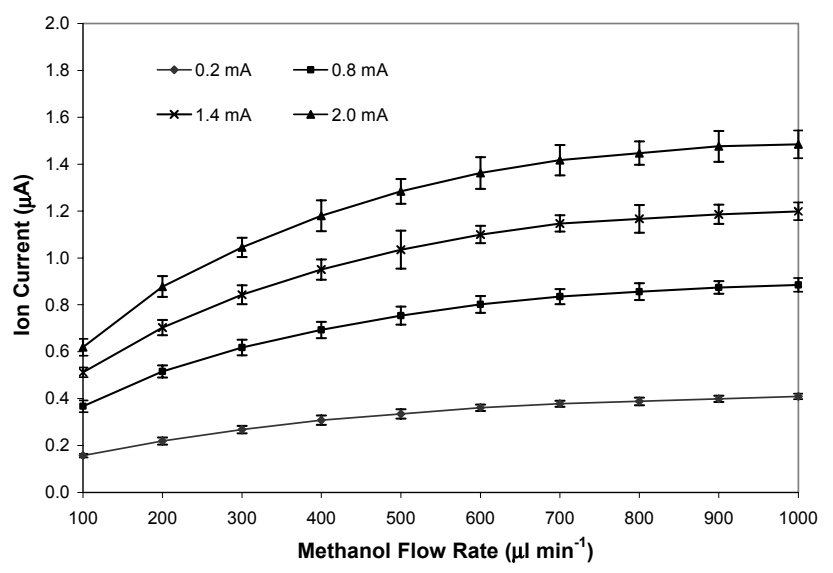

Figure 8. Plots of ion current versus methanol solvent flow rate, for several lamp current settings. The toluene dopant flow was set to $10 \%$ of the solvent flow.

number density of $\mathrm{S}^{*}\left(\mathrm{~cm}^{-3}\right)$. From the ratio of the rate of Penning ionization to the combined rates of all the processes involving $\mathrm{S}^{*}$, we can then express another term for ionization efficiency, $\phi_{S}$, this time for Penning ionization of the dopant by $S^{*}$ :

$$
\phi_{S}=\frac{k_{7} \cdot n_{D}}{k_{7} \cdot n_{D}+k_{8}+k_{9}+k_{10} \cdot n_{S}+k_{11} \cdot n_{N_{2}}}
$$

Thus, the expression for the total primary rate of dopant ionization ultimately becomes

$$
R_{P}=\phi_{T} \cdot I_{D}+\phi_{S} \cdot I_{S}
$$

where $\phi_{S}$ may be an increasing function of the dopant flow rate. This is one explanation for how the decline in ionization rate at a given toluene flow is less severe than predicted when solvent is added.

The last of the experimental results are presented in Figure 8, which shows plots of ion current versus methanol solvent flow rate, for several lamp current settings, all obtained with the toluene dopant flow set to $10 \%$ of the solvent flow (as is recommended for DAAPPI applications [22]). For each lamp current setting, the ion current initially increases with methanol flow rate, though the slopes of the plots appear to be reduced at higher flows. The latter observation is at least in part an artifact of a limitation in the measurement process. Control experiments have shown that as the methanol flow is increased above about $500 \mu \mathrm{l} \mathrm{min}^{-1}$ the measured signal decreases below the true $i_{S A T}$, because complete charge collection does not occur at $V=3 \mathrm{kV}$. The presence of large quantities of methanol in the vapor evidently affects the mobility of the photoelectrons. In any event, these results indicate that, for a fixed ratio of dopant-to-solvent, no reduction in $R_{P}$ occurs as the solvent flow is increased. Thus, quenching of excited-state precursors to the dopant ions, leading to a reduction in the primary rate of ionization, is not the mechanism responsible for the decline in sensitivity of the DA-APPI method with increased solvent flow.

Figure 9 shows plots of experimental ion current and calculated $I_{D} / I_{0}$ versus methanol solvent flow, with the toluene dopant flow set to $10 \%$ of the solvent flow, again for lamp current $=0.8 \mathrm{~mA}$. The same scaling factor has been used as for Figures 5 and 7 (i.e., $C=2.76$ $\mu \mathrm{A})$. From our previous discussion, we expect that the experimental and theoretical plots may only coincide at the lowest dopant and solvent flow rates, since we have argued that Penning ionization, via both $\mathrm{D}^{*}$ and $\mathrm{S}^{*}$, may increasingly occur as the dopant and solvent flows are increased. The actual result is in agreement with this expectation. At methanol flow $=100 \mu \mathrm{lmin}^{-1}$, the lowest flow tested, the experimental ion current is close to the theoretical value obtained from the product of $I_{D} / I_{0}$ times $C$. As the methanol flow is increased, the experimental plot increasingly diverges from the flat theoretical plot (except for at the highest methanol flows, where the experimental ion currents may be too low), in accordance with our hypotheses regarding Penning ionization. Thus, the results in Figure 9 support our speculations that our initial theoretical model and our empirically-determined $C$ value are accurate under low dopant and solvent flow conditions, and that an additional, previously ignored ionization mechanism-Penning ionization from excited-state intermediates-becomes active when the dopant and solvent flows are increased.

\section{Conclusions}

The experimental results indicate the following: with or without solvent, the primary rate of dopant ionization, $R_{P}$, can be increased by raising the dopant flow rate and/or the lamp current, and, when the dopant flow is maintained at a fixed percentage relative to the solvent, $R_{P}$ increases with the solvent flow rate. Thus, solvent-

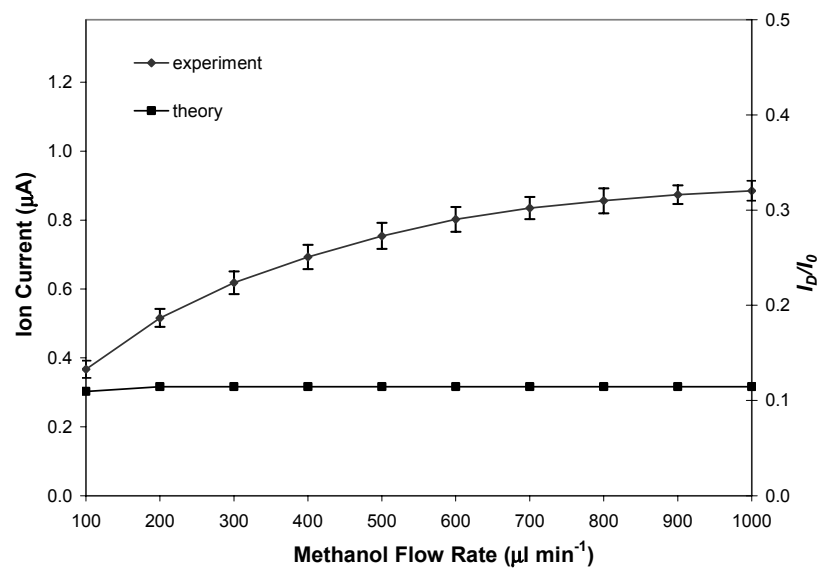

Figure 9. Plots of ion current (experiment) and calculated $I_{D} / I_{O}$ (theory) versus methanol solvent flow rate. The toluene dopant flow was set to $10 \%$ of the solvent flow. For the experimental data, lamp current $=0.8 \mathrm{~mA}$. The scaling factor, $C$, for the two vertical axes is $2.76 \mu \mathrm{A}$. 
induced collisional quenching of $\mathrm{D}^{*}$ does not limit $R_{P}$, and some other mechanism(s) must be responsible for the decline in DA-APPI sensitivity with increased solvent flow (see reference [9]).

There is some evidence that Penning ionization via excited-state dopant and solvent intermediates also contributes to the primary rate of dopant ionization in DA-APPI. At this juncture, however, the hypotheses regarding Penning ionization are speculative, and further study is certainly required to verify them.

\section{Acknowledgments}

The authors acknowledge the Natural Sciences and Engineering Research Council of Canada and the University of British Columbia for financial support.

\section{References}

1. Robb, D. B.; Covey, T. R.; Bruins, A. P. Atmospheric Pressure Photoionization: An Ionization Method for Liquid Chromatography-Mass Spectrometry. Anal. Chem. 2000, 72, 3653-3659.

2. Raffaelli, A.; Saba, A. Atmospheric Pressure Photoionization Mass Spectrometry. Mass Spectrom. Rev. 2003, 22, 318-331.

3. Hsieh, Y.; Wang, G. Integration of Atmospheric Pressure Photoionization Interfaces to HPLC-MS/MS for Pharmaceutical Analysis. Am. Pharmaceut. Rev. 2004, 7, 88-93.

4. Robb, D. B.; Covey, T. R.; Bruins, A. P. Atmospheric Pressure Photoionization: A New Ionization Technique of LC/MS. Proceedings of the 48th ASMS Conference on Mass Spectrometry and Allied Topics; Long Beach, CA, June, 2000.

5. Yang, C.; Henion, J. Atmospheric Pressure Photoionization Liquid Chromatographic-Mass Spectrometric Determination of Idoxifene and Its Metabolites in Human Plasma. J. Chromatogr. A 2002, 970, 155-165.
6. Hsieh, Y.; Merkle, K.; Wang, G.; Brisson, J.-M.; Korfmacher, W. A. High-Performance Liquid Chromatography-Atmospheric Pressure Photoionization/Tandem Mass Spectrometric Analysis for Small Molecules in Plasma. Anal. Chem. 2003, 75, 3122-3127.

7. Wang, G.; Hsieh, Y.; Korfmacher, W. A. Comparison of Atmospheric Pressure Chemical Ionization, Electrospray Ionization, and Atmospheric Pressure Photoionization for the Determination of Cyclosporin A in Rat Plasma. Anal. Chem. 2005, 77, 541-548.

8. Kauppila, T. J.; Bruins, A. P.; Kostiainen, R. Effect of the Solvent Flow Rate on the Ionization Efficiency in Atmospheric Pressure Photoionization-Mass Spectrometry. J. Am. Soc. Mass Spectrom. 2005, 16, 1399-1407.

9. Robb, D.; Blades, M. Effects of Solvent Flow, Dopant Flow, and Lamp Current on Dopant-Assisted Atmospheric Pressure Photoionization (DA-APPI) for LC-MS. Ionization via Proton Transfer. J. Am. Soc. Mass Spectrom. 2005, 16, 1275-1290.

10. Sevcik, J.; Krysl, S. A Photoionization Detector. Chromatographia 1973, 6, 375-380.

11. Freedman, A. N. The Photoionization Detector. Theory, Performance, and Application as a Low-Level Monitor of Oil Vapor. J. Chromatogr. 1980, 190, 263-273.

12. De Wit, J. S. M.; Jorgenson, J. W. Photoionization Detector for OpenTubular Liquid Chromatography. J. Chromatogr. 1987, 411, 201-212.

13. http://www.cathodeon.com/articles/article1.htm

14. Tanaka, Y. Absorption Spectrum of Nitrogen in the Region from 1075 to 1650 Å. J. Opt. Soc. Am. 1955, 45, 663-664.

15. http://webbook.nist.gov/chemistry.

16. Loeb, L. B. Electrical Conduction in Gases Below Ionization by Collision. In Basic Processes of Gaseous Electronics; University of California Press: Berkeley, CA, 1955, pp 597-646.

17. Lovelock, J. E. Ionization Methods for the Analysis of Gases and Vapors. Anal. Chem. 1961, 33, 162-178.

18. Bruins, A. P. Mass Spectrometry with Ion Sources Operating at Atmospheric Pressure. Mass Spectrom. Rev. 1991, 10, 53-77.

19. SCIEX On-Line Documentation. APCI Heated Nebulizer Inlet Manual. 1997.

20. Shaw, D. A.; Holland, D. M. P.; MacDonald, M. A.; Hayes, M. A.; Shpinkova, L. G.; Rennie, E. E.; Johnson, C. A. F.; Parker, J. E.; von Niessen, W. An Experimental and Theoretical Study of the Spectroscopic and Thermodynamic Properties of Toluene. Chem. Phys. 1998, 230, 97-116.

21. Burton, G. R.; Chan, W. F.; Cooper, G.; Brion, C. E. Absolute Oscillator Strengths for Photoabsorption $(6-360 \mathrm{eV})$ and ionic photofragmentation $(10-80 \mathrm{eV})$ of methanol. Chem. Phys. 1992, 167, 349-367.

22. SCIEX PhotoSpray Ion Source Operator's Manual. December 2002. 